\title{
31P-Topical Nuclear Magnetic Resonance (31P-TMR) Studies of Cardiotoxic Effects of 5-Fluorouracil (5-FU) and 5'-Deoxy-5-Fluorouridine (5'-DFUR)
}

\author{
Hirokuni TAMATSU, Mikio NAKAZAWA, Shoichi IMAI \\ and ${ }^{*}$ Hiroshi WATARI \\ Department of Pharmacology. Niigata University School of Medicine, Niigata 951. Japan \\ *Okazaki National Research Institutes. National Institute for Physiological Sciences, \\ Okazaki 444, Japan
}

Accepted December 19, 1983

\begin{abstract}
Using the ${ }^{31} \mathrm{P}$-topical nuclear magnetic resonance method (TMR), an attempt was made to determine the myocardial high-energy phosphate compounds (HEP) contents under in situ conditions in closed-chest animals, and the effects of opening the thorax on the myocardial energy metabolism were studied comparing the cardiotoxic effects of 5-fluorouracil (5-FU) in closed-chest and open-chest animals. It was found that the depletion of myocardial HEP produced by 5-FU was much more marked in open-chest animals than in closed-chest ones, indicating the necessity of conducting the experiments in closed-chest animals for the proper evaluation of the cardiotoxicity of certain types of compounds. Therefore, the cardiotoxicity of a prodrug of 5 -FU was assessed in closed-chest animals, and it was found to be less cardiotoxic than $5-\mathrm{FU}$.
\end{abstract}

For technical reasons, previous studies on the effects of various agents on the highenergy phosphate compounds (HEP) of the ventricular myocardium have been done either in isolated hearts or in open-chest animals. In view of the possible deleterious effects of opening the thorax on the myocardial energy metabolism, an attempt was made to determine the myocardial HEP contents under in situ conditions in closedchest animals with the newly-developed topical nuclear magnetic resonance method (TMR). This is a method that enables the acquisition of ${ }^{31} \mathrm{P}-\mathrm{NMR}$ spectra from a selected, localized area lying deep within the animal body. Using the same method, the HEP contents were also determined in openchest animals. As the determination of the absolute level of the HEP is rather difficult, though not impossible, with the nuclear magnetic resonance technique, the effects of opening the thorax on myocardial energy metabolism were studied comparing the cardiotoxic effects of 5 -fluorouracil in closed- chest animals with those in open-chest animals. As reported in a previous paper, 5-FU is a compound which produces a depletion of HEP after a rather long latent period (1).

The effects of a prodrug of 5-FU were also studied in closed-chest animals.

\section{Materials and Methods}

Male guinea pigs weighing around $400 \mathrm{~g}$ were anesthetized with urethane $(1.5 \mathrm{~g} / \mathrm{kg}$. i.p.). The myocardial HEP level was continuously monitored with the ${ }^{31} \mathrm{P}-\mathrm{NMR}$ technique using a TMR-32/200 (Oxford Research Systems) in the animals in a prone position under artificial respiration with room air $(80 / \mathrm{min}$, tidal volume of $1 \mathrm{ml} / 100 \mathrm{~g})$. TMR is a method that enables the acquisition of $31 \mathrm{P}-\mathrm{NMR}$ spectra from a selected. localized area lying deep within the animal body. This is done by modifying the main magnetic field using static-field gradients (2. 3). In this experiment, the profile of the magnetic field was adjusted in such a way 
that high resolution ${ }^{31} \mathrm{P}-\mathrm{NMR}$ signals can be obtained only from a spherical area of $2 \mathrm{~cm}$ diameter. ${ }^{31} \mathrm{P}-\mathrm{NMR}$ spectra were obtained with a $2 \mathrm{~cm}$ diameter surface coil by applying $10 \mu \mathrm{sec}$ pulses $\left(30^{\circ}-40^{\circ}\right.$ pulses) at $1 \mathrm{sec}$ intervals to reduce quantitation artifacts due to saturation and accumulating 600 transients (total acquisition time: $10 \mathrm{~min}$ ). Beginning with $10 \mathrm{~min}$ before administration of the drugs, this operation was repeated every 20 min. Resonance peaks were traced, and areas under each peak were determined by cuttingand-weighing the traced resonance peaks and expressed as percent of the respective resonance areas before drug administration. To normalize the data obtained from individual animals, methylene diphosphonate (MDP) was used as an external standard. The capillary tube containing a $1 \mathrm{M}$ solution of this compound was placed in front of the anterior chest wall. The standard limb lead II ECG was recorded before and after this operation (Fig. 1). In experiments conducted in closed-chest animals, pectoral muscles were removed as much as possible. In openchest animal experiments, the chest wall in front of the heart was removed while taking

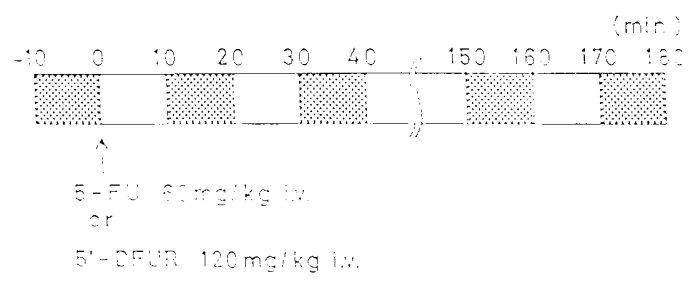

Fig. 1. Experimental protocol. period of accumulation of 600 transients (total acquisition time: $10 \mathrm{~min}$ ) by applying $10 \mu$ sec pulses at 1 sec intervals.
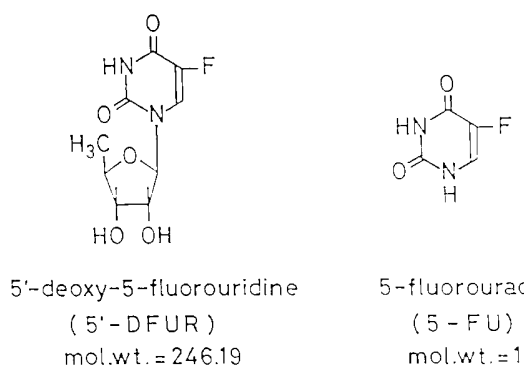

$$
\begin{aligned}
& 5 \text {-fluorouracil } \\
& (5-F \cup) \\
& \text { mol.wt }=130.08
\end{aligned}
$$

Fig. 2. Chemical structures of 5-FU and $5^{\prime}$-DFUR. every caution to avoid bleeding. Drugs were administered into the cervical vein. The doses used were $60 \mathrm{mg} / \mathrm{kg}$ for $5-\mathrm{FU}$ (mol. $w t .=130.08)$ and $120 \mathrm{mg} / \mathrm{kg}$ for $5^{\prime}$-DFUR (mol. wt. $=246.19)$ (Fig. 2). These were the doses presumed to exhibit the carcinostatic effect of a similar magnitude on neoplastic tissues (4). The 5-FU provided by Kyowa Hakko Kogyo was a $250 \mathrm{mg} / 5 \mathrm{ml}$ aqueous solution which contained $423.5 \mathrm{mg}$ of trishydroxyaminomethane $(0.7 \mathrm{M})$ as solvent. $5^{\prime}$-DFUR, which was provided by Nippon Roche K.K., was dissolved in $0.7 \mathrm{M}$ trishydroxyaminomethane. All values were expressed as means \pm standard errors. Statistical significance of a difference between two means was determined by Student's $t$-test.

\section{Results}

Figure 3 shows typical ${ }^{31} \mathrm{P}-\mathrm{NMR}$ spectra taken from the heart in closed-chest animals

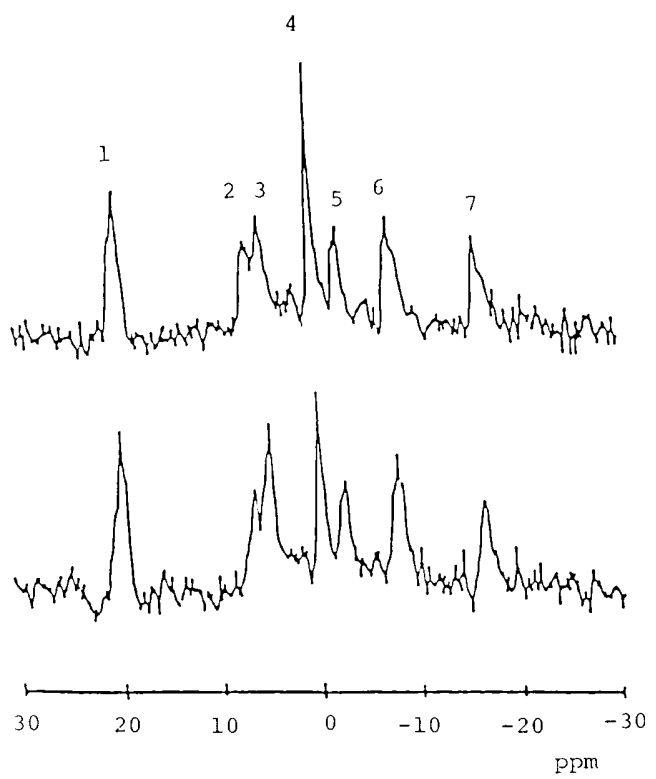

Fig. 3. A representative record of ${ }^{31} \mathrm{P}-\mathrm{NMR}$ spectra showing the effects of $5-F U$. The upper record was obtained before the drug administration and the lower, from 170 to $180 \mathrm{~min}$ after the drug administration. Resonance peaks are: (1) methylene diphosphonate (external standard), (2) sugar phosphates and AMP. (3) inorganic phosphate, (4) creatine phosphate. (5) $\gamma$-phosphate of ATP and $\beta$-phosphate of ADP. (6) $\alpha$-phosphate of ATP and NAD ${ }^{++}$ $\mathrm{NADH}$. (7) $\beta$-phosphate of ATP. 
and the changes produced therein within $3 \mathrm{hr}$ after administration of 5-FU. The left-most peak is from the solution of 1 M MDP used as an external standard. The areas of creatine phosphate (CP) and ATP peaks and the CP/ATP ratio at the start of the experiments expressed as \% of the areas of MDP peaks used as external standard were $77.4 \pm 13.6 \%$ $(n=7), 60.7 \pm 8.3 \% \quad(n=7)$ and $1.26 \pm 0.13$ $(n=7)$, respectively. There was a marked decrease in the CP peak at 170-180 min after administration of 5 -FU. Figure 4 shows typical ECG changes. The S-T segment elevation appeared first, followed by the large T. Figure 5 summarizes the data obtained from open-chest and closed-chest
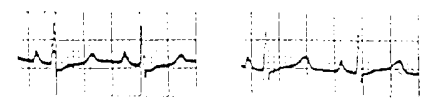

90

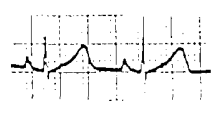

100
Fig. 4. A representative record of standard limb lead II ECG showing the effects of 5-FU. In this figure, numbers represent minutes after the drug administration. animals. Myocardial CP and ATP contents at the time of appearance of ECG changes were $80.1 \pm 13.9 \%(n=5)$ and $105.6 \pm 9.9 \%$ $(n=5)$, respectively, of the values before administration of 5-FU. In open-chest animals, the areas of CP and ATP peaks and the CP/ATP ratio were $58.0 \pm 7.7 \%(n=5)$, $59.2 \pm 7.8 \% \quad(n=5)$ and $0.98 \pm 0.07 \quad(n=5)$, respectively, at the outset. The depletion of myocardial HEP produced by 5-FU was much more marked in open-chest animals than in closed-chest ones (Fig. 5). The mortality of the animals was also higher in open-chest animals than in closed-chest ones. While there was no incidence of death during the entire experimental period of $3 \mathrm{hr}$ after administration of 5 -FU in closed-chest animals, death was observed in $40 \%(2 / 5)$ in open-chest animals (Fig. 5). Administration of tris-buffer, the solvent for 5-FU, to the open-chest animals resulted in no change in ECG changes or in HEP content (data not shown).

An equipotent carcinostatic dose of $5^{\circ}$ DFUR did not produce any change in the

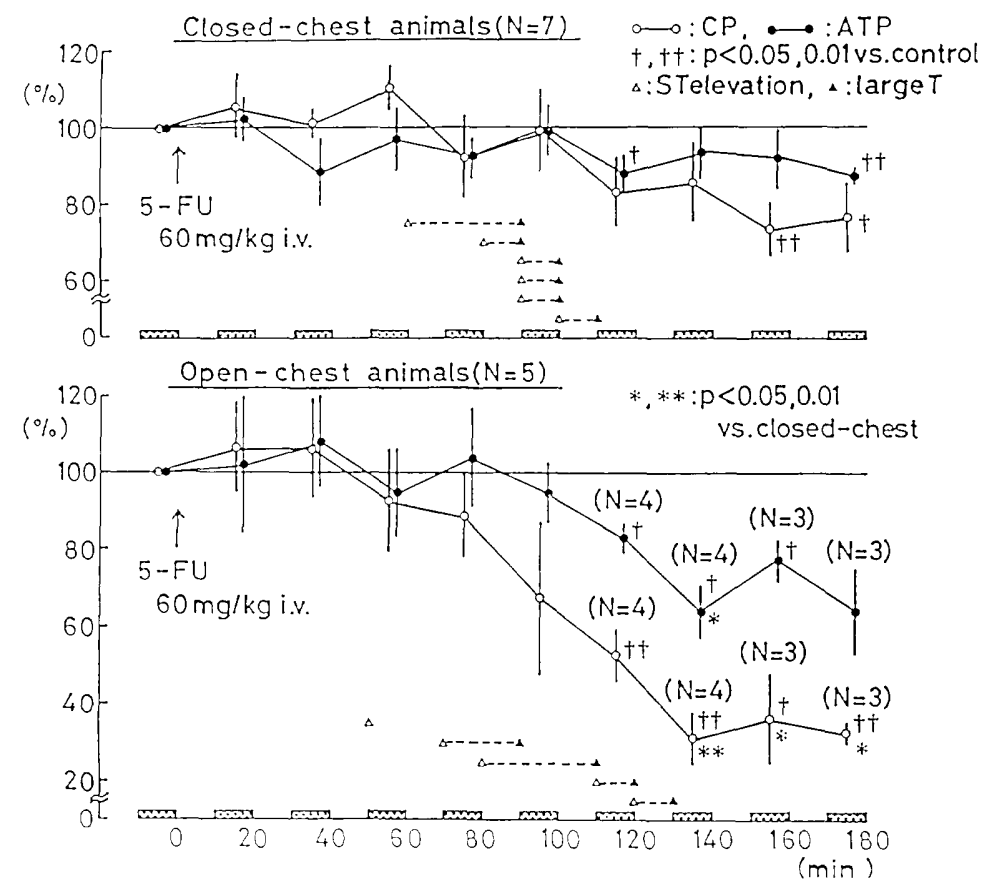

Fig. 5. The effects of 5-FU on myocardial HEP and ECG in open-chest guinea pigs, as compared with those in closed-chest animals. Each point represents the mean \pm S.E. 


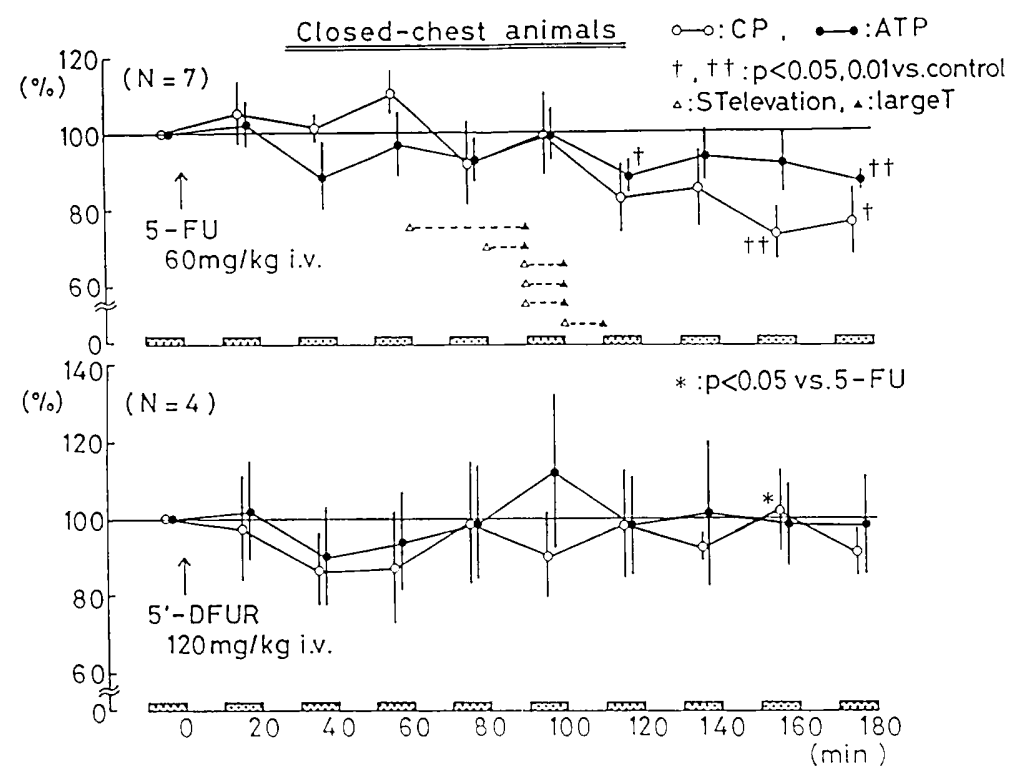

Fig. 6. The effects of 5-FU and 5'-DFUR on myocardial HEP and ECG in closed-chest guinea pigs. Each point represents the mean \pm S.E.

myocardial HEP contents (Fig. 6). There was also no changes in ECG over the period of $5 \mathrm{hr}$.

\section{Discussion}

All previous studies of myocardial energy metabolism have been done with isolated hearts or with open-chest animals. In this experiment, ${ }^{31} \mathrm{P}-\mathrm{TMR}$ spectroscopy was used to measure the myocardial HEP content in situ in the closed-chest animals in order to know the possible deleterious effects of opening the thorax on the myocardial HEP contents. To emphasize the effects, a cardiotoxic agent, 5-FU was used, which was shown in our previous study to produce a depletion of HEP (1). It was found that $5-\mathrm{FU}$ was much more cardiotoxic in open-chest animals than in closed-chest ones, indicating that opening the thorax causes an exaggeration of the cardiotoxic effects of 5-FU. The difference cannot be due to the contamination of $\mathrm{CP}$ and ATP signals of the residual pectoral muscles in closed-chest animals. In closed-chest animals, the initial CP peak areas were about $80 \%$ and the initial ATP peak areas were about $60 \%$ of the areas of the MDP peak, while both CP and ATP peak areas were about $60 \%$ in open-chest animals, giving a CP/ATP ratio of about 1.0 in open-chest animals and about 1.3 in closed-chest ones. In skeletal muscles, the CP/ATP ratio was about 2.7 (data not shown). Suppose there is contamination from residual pectoral muscles, then the $\mathrm{CP} / \mathrm{ATP}$ ratio of about 1.3 obtained in closed-chest animals means that about $40 \%$ of the $C P$ and about $20 \%$ of the ATP are from pectoral muscles. These portions of the HEP may not decrease under the influence of 5-FU. However, this cannot fully explain the difference noted relative to the changes in HEP produced by 5 -FU between the closedchest and open-chest animals. Furthermore, there was no incidence of death during $3 \mathrm{hr}$ after administration of 5-FU in the closedchest group. while death was observed in $40 \%(2 / 5)$ of the open-chest group, suggesting that there is a real difference in the severity of the myocardial derangement between open- and closed-chest animals. It is possible that the changes produced in the intrathoracic pressure by opening of the chest and the consequent changes in the reserve of cardiac function have something to do with the difference. 
In the present study, the appearance of S-T segment elevation preceded a significant depletion of myocardial HEP. As shown in Fig. 4, the shape of the elevation of the S-T segment was concave, a change characteristic of acute pericarditis (5), suggesting that the S-T segment elevation observed was caused by the injury of the superficial myocardium, resulting in production of the current of injury. Since HEP content was determined in whole myocardium, a significant depletion of myocardial HEP in the superficial myocardial cells, even if it was present, may have escaped detection.

It was demonstrated in the present experiments that, unlike 5-FU, equipotent carcinostatic doses of $5^{\prime}$-DFUR did not produce any cardiotoxic effects during the entire experimental period of $5 \mathrm{hr}$. $5^{\prime}$-DFUR is a prodrug of 5-FU that is claimed to exert its effects selectively on neoplastic tissues after being converted to 5 - FU by pyrimidine nucleoside phosphorylase which is present more abundantly in neoplastic tissues than in normal tissues including the heart (4). The results of the present study are consonant with such a mode of action of $5^{\prime}$-DFUR.

\section{References}

1 Matsubara, I., Kamiya, J. and Imai, S.: Cardiotoxic effects of 5-fluorouracil in the guinea pig. Japan. J. Pharmacol. 30, 871-879 (1980)

2 Gordon, R.E.: Topical magnetic resonance. Biosci. Rep. 2, 701-706 (1982)

3 Gordon, R.E., Hanley, P.E., Shaw, D., Gadian, D.G., Radda, G.K., Styles, P., Pore, P.J. and Chan, L.: Localization of metabolites in animals using $31 \mathrm{P}$ topical magnetic resonance. Nature 287, 736-738 (1980)

4 Ishitsuka, H., Miwa M., Takemoto, K., Fukuoka, K., Itoga, A. and Maruyama, H.B.: Role of uridine phosphorylase for antitumor activity of $5^{\prime}$-deoxy5-fluorouridine. Gann 71, 112-123 (1980)

5 Surawicz, B. and Lasseter, F.K.C.: Electrocardiogram in pericarditis. Am. J. Cardiol. 26, 471-474 (1970) 